\title{
Gobierno electrónico como tecnología de inclusión social. Reflexiones desde el Trabajo Social
}

\author{
Helder Binimelis-Espinoza \\ Universidad Católica de Temuco (UCT)
}

Gobierno electrónico como tecnología de inclusión social. Reflexiones desde el Trabajo Social Resumen: El gobierno electrónico es una herramienta de política pública aparentemente inevitable que transforma la acción del gobierno y la práctica profesional del trabajo social. Sobre la base del desafío ético y político que implica la práctica profesional del trabajo social, se presenta un análisis crítico sobre las interpretaciones del gobierno electrónico como una herramienta neutral o ideológicamente determinada. También se discute su relación con el poder y sus efectos negativos sobre la ciudadanía. Finalmente, se propone como alternativa para el trabajo social pensar el gobierno electrónico como una tecnología social orientada a la inclusión social y a la formación de una ciudadanía socio-técnica capaz de comprender los efectos de la tecnología en la sociedad y en su vida.

Palabras clave: Trabajo Social. Gobierno Electrónico. Inclusión Social.

\section{O governo eletrônico como tecnologia de inclusão social: reflexões a partir do Serviço Social}

Resumo: O governo eletrônico é uma ferramenta de política pública aparentemente inevitável que transforma a ação do governo e a prática profissional do Serviço Social. Este trabalho apresenta uma análise crítica das interpretações sobre governo eletrônico como uma ferramenta neutra ou ideologicamente determinada, considerando os desafios éticos e políticos envolvidos na prática profissional do Serviço Social. Também discute sua relação com o poder e seus efeitos negativos sobre a cidadania. Finalmente, propõe como alternativa para o Serviço Social pensar o governo eletrônico como uma tecnologia social orientada para a inclusão social e a formação de uma cidadania sócio-técnica capaz de compreender os efeitos da tecnologia na sociedade e na vida.

Palavras-chave: Serviço Social. Governo eletrônico. Inclusão social.

\section{Electronic Government as Technology for Social Inclusion: Reflections based on Social Work}

Abstract: Electronic government is an apparently inevitable public policy tool that transforms government action and the professional practice of social work. This paper presents a critical analysis of interpretations of electronic government as a neutral or ideologically determined tool, considering the ethical and political challenges involved in the professional practice of social work. It also discusses its relationship with power and its negative effects on citizenship. Finally, it proposes that social work consider electronic government as a social technology that is oriented towards social inclusion and to formation of socio-technical citizenship that is capable of comprehending the effects of technology in society and in life.

Keywords: Social Work. Electronic Government. Social Inclusion. 


\section{Introducción}

Desde fines del siglo XX, y particularmente desde la publicación del Informe del Milenio (ANNAN; NACIONES UNIDAS, 2000), la utilización de tecnologías de información y comunicación (TIC) se ha convertido en una tendencia gubernamental asociada con la mejora de procesos administrativos y nuevas formas de vinculación con la ciudadanía. Se conoce como gobierno electrónico al conjunto de políticas públicas orientadas a la utilización de TIC en diversos ámbitos de acción gubernamental, con una creciente presencia en Latinoamérica y el resto del mundo (CONFERENCIA IBEROAMERICANA DE ADMINISTRACIÓN PÚBLICA Y REFORMA DEL ESTADO, 2007; NACIONES UNIDAS, Departamento de Asuntos Económicos y Sociales, 2003, 2004, 2005, 2008, 2010). En este contexto, los objetivos planteados aquí son desarrollar una interpretación crítica respecto a la comprensión social del gobierno electrónico como tecnología y distinguir los desafíos que ello implica para el trabajo social en su relación con la ciudadanía y la permanente necesidad de democratizar la sociedad.

Las perspectivas teóricas respecto al gobierno electrónico lo sitúan como un campo de estudios interdisciplinar, siendo difícil establecer una definición consensuada del término. Gil-García y Luna-Reyes (2006, p. 637-638) plantean que existen al menos tres aproximaciones para definir al gobierno electrónico. La primera es la distinción de los tipos de actividad que pueden efectuarse electrónicamente, donde Cunha y Miranda (2013, p. 545) mencionan la administración, los servicios, y la democracia electrónica. La segunda aproximación es a partir de los actores involucrados, en que se identifican cuatro interacciones clave: gobiernociudadanos; gobierno-empresas; gobierno-otros gobiernos y gobierno-empleados (GIL-GARCÍA; LUNAREYES, 2006, p. 637). La última aproximación es a partir de la evolución en su implementación, es decir, el grado de avance respecto al tipo de actividades que se pueden desarrollar con la información gestionada electrónicamente, la que va del simple catalogamiento de información hasta procesos complejos de integración vertical y horizontal entre servicios públicos.

Sin embargo, estos enfoques resultan insuficientes para abordar las diversas tensiones en los procesos de implementación de políticas públicas de gobierno electrónico, tales como: barreras administrativas desarrolladas tanto por gobiernos como por funcionarios (AIKINS; KRANE, 2010); deficiencias administrativas y de gestión en la interacción con la ciudadanía (BIGDELI; KAMAL; DE CESARE, 2013); y limitados efectos sobre participación ciudadana y la democracia (SANDOVAL-ALMAZAN; GIL-GARCIA, 2012; RAUPP; PINHO, 2013). A lo anterior se suma la exclusión tecnológica y la brecha digital en la medida en que las diferencias sociales, culturales, y de integración económica, producen distinciones en el impacto que las TIC tienen sobre los diversos grupos sociales (BINIMELIS, 2008).

Es por ello que para plantear una evaluación en este ámbito de política pública, y su vinculación con el trabajo social parece relevante dar un paso previo, esto es, distinguir las concepciones de tecnología que subyacen tras la implementación de políticas de gobierno electrónico, y cómo a partir de ellas es posible (o no) reducir la tensión entre avances tecnológicos y exclusión social.

Se acotará la discusión a partir de los aportes de la teoría crítica de la tecnología (FEENBERG, 2012), y algunas de las propuestas más relevantes en el área planteadas desde América Latina: la adecuación sociotécnica y su discusión sobre tecnología social (DAGNINO; BRANDÃO; NOVAES, 2004; THOMAS, 2009a). A partir de ello, es que se propone el desafío de pensar el gobierno electrónico desde el trabajo social como una tecnología social, y el aporte que puede hacer el trabajo social en relación a la construcción de una ciudadanía socio-técnica, proponiendo una discusión sobre las siguientes preguntas: ¿cuál es el papel de análisis crítico que le corresponde al trabajo social en relación a las políticas públicas de gobierno electrónico? ¿puede asumir el trabajo social el desafío de la formación digital de la ciudadanía? Una cuestión previa es, entonces, abordar el vínculo entre la tecnología y sus consecuencias sociales.

\section{Sobre las consecuencias sociales de la tecnología}

En esta sección se desarrollará una crítica a tres cuestiones centrales en la comprensión de la tecnología: la neutralidad, el determinismo y el poder. Además, a partir del desarrollo de una interpretación alternativa, la teoría crítica de la tecnología, se examinan estas cuestiones en relación al gobierno electrónico.

\subsection{Neutralidad o determinismo, y el problema del poder tecnológico}

Se entenderá por neutralidad la posibilidad de crear un instrumento técnico que se adapte a los fines de diversos actores sociales, sin importar sus experiencias pasadas y sus expectativas futuras. Ello implica que 
una tecnología, como el gobierno electrónico, puede ser exportada a cualquier contexto social, valórico y cultural, donde responderá eficientemente a las tareas que allí se le asignen. Según señala Feenberg (1991), ésta es la concepción predominante de la tecnología asociada a los procesos de modernización y desarrollo.

Una segunda cuestión es si las consecuencias del uso de las tecnologías modernas implican determinismo: ciertos futuros definidos a partir de opciones ideológicas sobre la tecnología, que pueden ser positivas o negativas. Por una parte, quienes creen en un futuro donde la tecnología ayudará a solucionar los principales problemas sociales, económicos y ambientales que hoy nos afligen (nuevamente como una interpretación dominante en relación a los procesos de modernización y desarrollo). Por otra, quienes ven en la tecnología una condena, en la que nuestro destino inevitable es convertirnos en simples engranajes de una maquinaria fuera de cualquier tipo de control racional, que puede terminar remplazándonos en la mayoría de las áreas productivas (interpretación que habitualmente desarrollan las perspectivas críticas al capitalismo).

Sin embargo, estas representaciones son parciales ya que implican visiones sesgadas de las consecuencias de la tecnología sobre la sociedad. Según expresa Feenberg (1991), estos modelos son dualistas (lo tomas o lo dejas) y se orientan a fijar límites sobre qué se puede o no hacer con tecnología, y como esos límites implican visiones morales que no se corresponden con su origen racional-técnico, en vez de transformar la tecnología tienden a prohibirla o limitarla.

Neutralidad y determinismo son, en realidad, componentes de un proceso tecnológico más complejo al que se denomina instrumentalización: "La teoría de la instrumentalización sostiene que la tecnología debe ser analizada en dos niveles: el nivel de nuestra original relación funcional con la realidad y el nivel del diseño y la implementación.” (FEENBERG, 2005, p. 112).

La funcionalidad entendida como instrumentalización primaria, momento en que se detectan propiedades racionales y eficientes que son separadas de sus contextos para convertirse en objetos técnicos manipulables. Aunque en principio esto pueda parecer neutralidad, la instrumentalización primaria siempre estará en una tensión dialéctica con los procesos de diseño e implementación, entendidos como instrumentalizaciones secundarias en las que los objetos técnicos son revinculados con los ambientes naturales y sociales donde serán utilizados.

El diseño y la implementación nunca son neutrales, ya

El diseño y la implementación nunca son neutrales, ya que responden a las diversas formas en que se organiza una sociedad en torno a cuestiones económicas, políticas y culturales. Por ello, aunque una tecnología se desarrolle a partir de principios racionales, su creación y uso van a depender de intereses y juegos de poder entre creadores $y$ usuarios. que responden a las diversas formas en que se organiza una sociedad en torno a cuestiones económicas, políticas y culturales. Por ello, aunque una tecnología se desarrolle a partir de principios racionales, su creación y uso van a depender de intereses y juegos de poder entre creadores y usuarios.

En el contexto del actual proceso de globalización, el cambio tecnológico, y especialmente la adopción de TIC, ocupan una posición central en el espacio económico capitalista. Si asumimos que las tecnologías no son neutrales, entonces, son los intereses y necesidades del capital los que se ponen de manifiesto en el diseño e implementación desarrollados en la instrumentalización secundaria. Según expresan Dagnino, Brandão y Novaes (2004, p. 45):

Esa situación permite entender el modo específico por el cual se da un conflicto social en la esfera técnica: si alternativas técnicamente comparables tienen implicaciones distintas en términos de distribución de poder, y si ocurre alguna disputa entre trabajadores y capitalistas (o sus representantes técnicos, los ingenieros), tiende a ser escogida aquella que favorece el control del proceso por estos últimos.

Feenberg plantea que el ejercicio del poder aplicado a la tecnología produce un estrechamiento, una deformación de la estructura social (y natural) de intereses y necesidades que habría que tomar en cuenta al momento de diseñar un artefacto técnico, generando con ello "sufrimiento" a los seres humanos y "daño al medio ambiente natural” (FEENBERG, 2005, p. 111). Más específicamente, son dos las formas de poder que predominan en el diseño de objetos tecnológicos en las sociedades capitalistas. Por una parte, la necesidad de control jerárquico que surge a partir de la forma en que se ha organizado el proceso de producción capitalista; 
y por otra parte, la orientación al consumo, la comprensión de la tecnología desde el fetichismo de la mercancía, que humaniza procesos y objetos técnicos y cosifica al ser humano debido a la forma en que se ha organizado el mercado capitalista (SANTOS, 2003).

De forma más específica, ello ocurre por medio de códigos técnicos, es decir, “[...] la realización de un interés bajo la forma de una solución técnicamente coherente a un problema [...]” (FEENBERG, 2005, p. 114), pero que implica un sesgo, una solución que siendo eficiente, responde a la forma en que se organiza el poder en la sociedad, cerrando las opciones a otras soluciones técnicamente más consideradas con el medio ambiente o con el sufrimiento humano. El poder en la tecnología, se ejerce, entonces, mediante la capacidad de no tomar en cuenta otras alternativas viables. Este proceso de control es denominado por Feenberg como autonomía operativa: “[...] la libertad del propietario o su representante para tomar decisiones independientes acerca de cómo manejar los negocios de la organización, sin tomar en cuenta los puntos de vista o los intereses de los actores subordinados y del entorno comunitario." (FEENBERG, 2005, p. 115).

Ello implica actuar a resguardo de las consecuencias (las que habitualmente son externalizadas para que las padezca el medio ambiente u otros seres humanos); e implica también la búsqueda de mantener las condiciones de ejercicio de poder y de autonomía en el futuro, de tal forma que se mantengan las condiciones de privilegio.

Más allá del espacio capitalista y la forma en que se vincula la tecnología con el poder económico institucionalizado, es importante precisar que, en el espacio de la ciudadanía, el lugar donde se desarrolla el gobierno electrónico, y donde se efectúa parte importante de la acción profesional del trabajo social, existen formas específicas de poder. Por una parte, la influencia ideológica del neoliberalismo y su comprensión del Estado y de las relaciones con la ciudadanía orientadas por un individualismo extremo que tiende hacia la despolitización, la mediatización y el consumismo; y por otra parte, las tendencias propias de la construcción política de nuestro contexto latinoamericano, en donde, el ejercicio del poder político se enmarca en herencias culturales clientelares, autoritarias, patriarcales y racistas (LARRAÍN, 2001). Es posible suponer que estas formas de poder afectan y se ponen de manifiesto en el ejercicio profesional del trabajo social tanto de forma directa como por medio de su instrumentalización tecnológica.

Una última cuestión a considerar es que dentro de la instrumentalización secundaria puede ocurrir una segunda tensión dialéctica entre las necesidades e intereses de los creadores de una tecnología, y las necesidades e intereses de los usuarios, quienes pueden desarrollar una instrumentalización secundaria alternativa. Estas formas de instrumentalizar están vinculadas con formas diferentes de ejercer el poder: por una parte, la acción estratégica y planificada institucionalmente de quienes desarrollan una tecnología en contextos capitalistas; y por otro lado, la confrontación táctica de los usuarios que se oponen a los límites preestablecidos institucionalmente, desarrollando marginalmente usos alternativos (FEENBERG, 2012; DE CERTEAU, 2007).

\subsection{Hacia una comprensión social del gobierno electrónico}

En relación a las cuestiones de neutralidad y determinismo aplicadas al gobierno electrónico, resulta relevante examinar los aportes de Heeks y Bailur (2007, p. 260), quienes al abordar la discusión sobre cienciatecnología aplicada al gobierno electrónico señalan que la interpretación dominante es de un “excesivo optimismo”, lo que produciría un sesgo respecto a la implementación y evaluación de estas políticas.

Por otra parte, Yýldýz y Saylam (2013) analizan discursos públicos sobre gobierno electrónico, destacando que las visiones positivas están fundamentalmente concentradas en actores institucionalizados como burócratas, políticos y periodistas.

Como señala Boeninger (2007), una de las cuestiones fundamentales en relación a la implementación de políticas públicas es el desarrollo de una agenda política, es decir, la construcción de un discurso que promueve la implementación o bloqueo de un determinado curso de acción política. En este sentido, es necesario preguntarse si los discursos políticos promovidos por organismos internacionales y gobiernos son poco realistas (excesivamente positivos) en relación a los efectos del gobierno electrónico sobre la sociedad.

En relación al vínculo entre poder y gobierno electrónico, es relevante señalar que en la instrumentalización secundaria, es decir, en el proceso de diseño e implementación de políticas de gobierno electrónico, existirían decisiones estratégicas que, por una parte, tienden a replicar las formas de poder capitalistas programadas en el código técnico del gobierno electrónico: aquellas orientadas al control de la ciudadanía y a la fetichización de la política; y por otra parte, se reproducirían las formas de poder preexistentes en el espacio de la ciudadanía. Como se había mencionado anteriormente, el neoliberalismo y la construcción cultural del poder político en un contexto social específico, se constituirían en el marco estratégico para la definición de políticas de gobierno electrónico que pueden potenciar un trabajo social controlador y excluyente. 
Según plantea Henman (2010), es posible detectar diversas formas de poder vinculadas al desarrollo de políticas de gobierno electrónico. El autor sitúa su análisis desde la comprensión del poder de Foucault, identificando la existencia de formas de poder disciplinario, que implican: "[...] que los individuos se gobiernen de acuerdo con los objetivos gubernamentales de las autoridades [...]” (HENMAN, 2010, p. 220); poder soberano orientado a la coerción; biopoder orientado a la regulación de la biología y el cuerpo; y formas de poder pastoral, orientadas a la conducción del "rebaño" ciudadano por el "pastor" que domina la información y los aspectos técnicos de la decisión política.

Junto con ello, identifica la existencia de formas de poder liberales y neoliberales. Las primeras implican un ejercicio del poder que transforma la búsqueda de libertad individual en programas y prácticas gubernamentales en las que entran en juego los procesos de elección, los deseos y las aspiraciones. Las segundas, que implican mecanismos indirectos de organizar la conducta, es decir:

[...] formas de inducción, mecanismos de incentivos, persuasión por parte de expertos y disciplina mediante vigilancia. Los individuos son considerados sujetos libres que pueden elegir sus formas de comportamiento, pero sus elecciones se realizan en entornos que han sido construidos por una serie de tácticas cuidadosamente calculadas, como indicadores de rendimiento y auditorías, incentivos y asesoramiento de expertos [...](HENMAN, 2010, p. 213). ${ }^{1}$

Es lo que ha sucedido, por ejemplo, en el ámbito ambiental, donde la solución política que se da a estos problemas, únicamente legitima como alternativas posibles las respuestas técnicas, no considerando los aportes de movimientos sociales y comunidades (FOLADORI; TOMMASINO, 2012).

Por otra parte, respecto al contexto cultural donde se implementan políticas de gobierno electrónico, Frei expresa que:

En las dimensiones funcionales, instrumentales y de eficacia, el gobierno electrónico muestra todo su potencial (todo el brillo del oro se podría decir), mientras que las dimensiones de interacción y acceso de información pública, sumado al centralismo, muestran la reproducción de una cultura política anterior a la instalación de las nuevas tecnologías. (FREI, 2007, p. 34).

¿Cuáles serían los componentes culturales del poder que orientarían al gobierno electrónico en América Latina? Por una parte, Frei destaca el centralismo que implicaría una distribución desigual de las políticas públicas, que, por medio de mecanismos de control burocrático, tenderían a potenciar la desigualdad y la exclusión de amplias zonas, aisladas y desconectadas. Por otra parte, destaca el papel que juegan las relaciones clientelares en la política latinoamericana, las que tendrían también su impacto en el ámbito del gobierno electrónico al reducir la ciudadanía al mero rol de clientes del Estado. Finalmente, y apoyado por la investigación previa del Programa de las Naciones Unidas para el Desarrollo - PNUD (2006), Frei expresa que en el examen de los procesos de interacción por vía electrónica entre ciudadanos y representantes políticos se ponen de manifiesto conductas clasistas y racistas.

En la discusión planteada hasta el momento se revela el estrechamiento de las opciones tecnológicas en el ámbito de la ciudadanía y el gobierno electrónico. Aparecen como dominantes las orientaciones al control jerárquico y la fetichización (previamente programadas en los códigos técnicos de las TIC), y las orientaciones propias del poder político en contextos específicos, es decir, de las opciones políticas en torno al neoliberalismo, y del desarrollo una cultura política latinoamericana.

Si la discusión quedara hasta aquí, parecería que se asume la opción de un determinismo tecnológico negativo, frente a lo cual, el trabajo social puede terminar convirtiéndose en una mera función mecánica. Sin embargo, es relevante destacar que lo que se ha discutido hasta el momento es la dimensión estratégica institucional del gobierno electrónico, y que todavía queda por explorar la dimensión táctica, desde donde:

Abrir la tecnología a una gama más amplia de intereses y preocupaciones podría llevar a su rediseño, para hacerla más compatible con los límites humanos y naturales relativos a la acción técnica. Una transformación democrática desde abajo puede acortar los ciclos de feedback provenientes de las deterioradas vidas humanas y la naturaleza y liderar una reforma radical de la esfera técnica. (FEENBERG, 2005, p. 111).

A continuación, vincularemos estas posibilidades de apertura democrática de la tecnología y del gobierno electrónico con las tecnologías sociales y el trabajo social. 


\section{Tecnologías sociales y Trabajo Social}

El análisis crítico elaborado por Guerra (2013, p. 41), caracteriza a la corriente central del trabajo social como orientada por una apropiación de lo social sin mediaciones teóricas o ideológicas, estableciendo relaciones a partir de sus manifestaciones inmediatas, cotidianas, sin abordar la cuestión de la génesis de los fenómenos sociales, los fundamentos de la política social o de su propia acción profesional.

En diversas publicaciones sobre Trabajo Social y TIC esta tendencia parece corroborarse. Por ejemplo, descripciones sobre la utilización de la internet of things ${ }^{2}$ o la gamification ${ }^{3}$ (GOLDKIND; WOLF, 2015), las que podrían ser incorporadas a las rutinas cotidianas de los trabajadores sociales debido a la neutralidad de sus principios técnicos. En varios otros trabajos sobre el tema de las TIC y el trabajo social (MISHNA et al., 2014; BAKER et al., 2014; GELMAN; TOSONE, 2010), las tecnologías aparecen exclusivamente como herramientas que otorgan roles preestablecidos a los trabajadores sociales y sus clientes, sin considerar la planificación estratégica, es decir, la definición de las mediaciones teóricas, políticas e ideológicas.

Frente a ello, algunos trabajos críticos proponen que las TIC no generan de forma automática efectos sociales positivos, sino que también pueden producir exclusión social (GUTIERREZ, 2012), y pueden alterar profundamente el rol desempeñado por los trabajadores sociales y los ciudadanos que requieren de su acción profesional mediada tecnológicamente (GILLINGHAM, 2014).

En el entendido de que el trabajo social implica no únicamente una experiencia práctica, sino también un proceso de comprensión epistemológica, ética y política de la sociedad (VIVERO, 2012), es que se discutirá a continuación el vínculo entre gobierno electrónico y tecnología social.

\subsection{Tecnologías sociales para la inclusión}

Una de las primeras nociones sobre tecnología social surge de las experiencias desarrolladas por Gandhi, en la lucha contra las injusticias sociales del sistema de castas, y en el proceso hacia la independencia de la India (DAGNINO; BRANDÃO; NOVAES, 2004, p. 19-20). Acciones técnicas simples como la extracción de sal del agua de mar o la utilización de la rueca de hilar para confrontar monopolios estatales y fuertes regulaciones de la producción industrial, dieron un nuevo sentido social y político a tecnologías aparentemente obsoletas.

La tecnología no es simplemente un producto dado, sino que es una construcción social, y por lo tanto, las condiciones contextuales y las relaciones de los actores sociales son fundamentales para comprender sus consecuencias. Se entiende que los actores sociales en sus respectivos contextos poseen diferentes capacidades y recursos para enfrentar los problemas que trae consigo la convivencia social, por lo que las soluciones tecnológicas nunca deben ser impuestas, sino democráticamente consensuadas, valorando tanto el conocimiento tradicional como el aporte de las tecnologías modernas.

Las tecnologías sociales permiten la adecuación contextual tanto de innovaciones capitalistas como de tecnologías aparentemente obsoletas usadas o que impliquen la recuperación de saberes no occidentales, ya que se orientan hacia la satisfacción de necesidades sociales y económicas de grupos y sujetos sociales concretos, facilitando en el proceso la introducción participativa de otras necesidades e intereses más allá de las meramente económicas.

Por ello, lo que se busca es la construcción de adaptaciones y alternativas tecnológicas para la solución de diversos problemas a los que no se da respuesta dentro de los sistemas tecnológicos y políticos tradicionales. En síntesis, como propone Thomas (2009b, p. 74), las tecnologías sociales serían: “[...] un modo de desarrollar e implementar tecnologías (de producto, proceso y organización), orientada a la generación de dinámicas de inclusión social y económica y desarrollo sustentable.”.

Thomas propone que las tecnologías sociales deben ser consideradas como parte de las estrategias de política pública para la inclusión social y el desarrollo, que los gobiernos latinoamericanos debieran potenciar. Junto con ello, las tecnologías sociales permitirían la constitución de una ciudadanía socio-técnica, es decir, una ciudadanía capaz de tomar democráticamente decisiones entorno a problemas sociales y alternativas tecnológicas:

[...] si existen alternativas tecnológicas y es posible elegir entre ellas, si los actores sociales pueden participar de estos procesos, y si las tecnologías constituyen la base material de un sistema de afirmaciones y sanciones que determina la viabilidad de ciertos modelos socioeconómicos, de ciertos regímenes políticos, así como la inviabilidad de otros, parece obvio que es imprescindible incorporar la tecnología como un aspecto fundamental de nuestros sistemas de convivencia democrática. (THOMAS, 2009b, p. 83).

Con ello se podría avanzar en uno de los principales déficit de las actuales políticas de gobierno electrónico, que como se ha indicado previamente, han resultado útiles en procesos de organización e interacción jerárquicos, 
pero con limitadas contribuciones en relación a la democratización y transparencia política al mantener una lógica de control top down en el proceso de formulación e implementación.

Desde el trabajo social se podrían retomar las preocupaciones previamente planteadas por Frei (2007), en torno a los problemas del vínculo entre democracia y el ámbito electrónico en América Latina. Es en torno a los problemas del centralismo, la burocratización, la falta de consideración por la desigualdad y la exclusión en la implementación de este tipo de políticas, en la construcción de relaciones clientelares, y en la falta de reconocimiento de las relaciones interculturales; que el trabajo social puede aportar en el desafío de distinguir las construcciones ideológicas y la forma en que ellas se convierten en diseños técnicos orientados por fines políticos.

No se propone, simplemente reemplazar un modelo jerárquico de control por otro democrático. Eso implicaría igualmente un estrechamiento de las posibilidades tecnológicas, cuando en realidad lo que se busca es la ampliación de las opciones. Una de las principales dificultades para ello no está en las decisiones de los gobiernos, sino en la ciudadanía. En realidad, quienes ejercen sus derechos ciudadanos activamente en la actualidad son una minoría; y aquellos ciudadanos con capacidades socio-técnicas que ejercen una ciudadanía activa son una minoría más reducida todavía. Lo que plantearemos a continuación es que el aporte del trabajo social puede ser fundamental en torno a la construcción de una relación socio-técnica democrática y participativa entre la ciudadanía y el gobierno electrónico.

\subsection{Trabajo Social y gobierno electrónico como tecnología social}

Se propone que un nuevo espacio de reflexión disciplinar y acción profesional para el trabajo social debe estar orientado a la búsqueda de ampliación de las necesidades sociales e intereses que se vinculan con el gobierno electrónico. En un campo de acción vinculado con la acción gubernamental, la política y el ejercicio de la ciudadanía, esas necesidades e intereses se asociarán con la búsqueda de ampliación de derechos sociales y políticos, donde el aporte del trabajo social puede ser fundamental. Se distinguen a continuación algunos desafíos para la investigación y el desempeño profesional.

Por una parte, la necesidad de alfabetizar digitalmente a los propios trabajadores sociales en la medida en que el vínculo con las TIC y con el gobierno electrónico adquiere cada día una presencia más relevante, constituyéndose en un desafío profesional que no puede enfrentarse adecuadamente sin las adecuadas mediaciones teóricas, éticas y políticas en torno a las consecuencias de la acción técnica. Ello permitiría una mayor injerencia en las políticas de gobierno electrónico, tanto en relación a su planificación y evaluación, como al rol preponderante que le corresponde al trabajo social en su implementación.

A partir de esa base, es posible asumir el desafío de la alfabetización digital de una ciudadanía socio-técnica, para afrontar las desiguales condiciones culturales, políticas y económicas, que excluyen de las TIC a una parte significativa de la sociedad, y en relación al complejo proceso de re-aprender a usar tecnologías buscando la ampliación de las opciones democráticas en vez de un uso centrado en el control y el consumo.

Solo trabajadores sociales y ciudadanos alfabetizados para un uso democrático del gobierno electrónico podrán colaborar efectivamente en la ampliación de las necesidades e intereses que se tomen en cuenta en la política pública. Surge como desafío desarrollar metodologías de acción profesional que permitan involucrar a los ciudadanos en las diversas etapas de planificación, implementación y evaluación de políticas públicas de gobierno electrónico. Si no es así, los ciudadanos están condenados a un rol de clientes pasivos y los trabajadores sociales, a un ejercicio mecánico de su profesión.

Finalmente, es relevante destacar que experiencias exitosas de gobierno electrónico y ciudadanía sociotécnica en otras partes del mundo (CASTELLS; HIMANEN, 2002), muestran que deben ampliarse las relaciones de interacción entre los diversos actores sociales involucrados en el ejercicio de la ciudadanía. No basta con un vínculo, habitualmente vertical entre ciudadanos y gobiernos, sino además debe procurarse la utilización de TIC para promover relaciones autónomas entre ciudadanos y organizaciones de la sociedad civil. El trabajo social puede contribuir en la creación y dinamización de plataformas digitales de comunicación e interacción entre diversos actores sociales.

\section{Palabras finales}

En las páginas previas se ha presentado una discusión crítica en torno a la aparentemente inevitable tendencia de implementar políticas de gobierno electrónico. Se ha señalado que para enfrentar las dificultades asociadas con este tipo de políticas públicas es fundamental que previamente se comprenda que tras toda tecnología hay una orientación ideológica y política, y que, debido a las diferencias sociales en la distribución del 
poder, el gobierno electrónico, puede ser principalmente encauzado hacia la satisfacción de las necesidades e intereses de los actores sociales dominantes.

Frente a ello se propone la opción de las tecnologías sociales en la búsqueda de un ejercicio democrático de ampliación de las necesidades e intereses que se tomen en cuenta al momento de implementar políticas públicas de gobierno electrónico. Es frente a esta preocupación que se propone que el trabajo social tiene como desafío la colaboración en la creación de las condiciones para que la ciudadanía puede ser adecuadamente ejercida de forma electrónica, en un contexto donde las tecnologías no son neutrales y donde las opciones tecnológicas no están inevitablemente programadas. Estos desafíos implican destacar la relevancia de la formación crítica en TIC en la educación de los trabajadores sociales, tanto como la capacidad de desarrollar acciones profesionales en la formación y acompañamiento de una ciudadanía socio-técnica orientada democráticamente.

\section{Referencias}

AIKINS, S. K.; KRANE, D. Are Public Officials Obstacles to Citizen-Centered E-Government? An Examination of Municipal Administrators’ Motivations and Actions. State \& Local Government Review, v. 42, n. 2, p. 87-103, 1 ago. 2010.

ANNAN, K.; NACIONES UNIDAS. Nosotros los Pueblos. La función de las Naciones Unidad en el siglo XXI. 2000. Organismo Internacional. Disponible en: <http://www.un.org/es/events/pastevents/millennium_summit/sg_report/full.htm>. Acceso en: 10 mayo. 2014.

BAKER, S. et al. Reimagining the Relationship between Social Work and Information Communication Technology in the Network Society. Australian Social Work, v. 67, n. 4, p. 467-478, 2014.

BIGDELI, A. Z.; KAMAL, M. M.; DE CESARE, S. Electronic information sharing in local government authorities: Factors influencing the decision-making process. International Journal of Information Management, v. 33, n. 5, p. 816-830, 2013.

BINIMELIS, H. La sociedad civil y las nuevas tecnologías de información y comunicación. In: LIVACIC, C. et al. Gestión y Organizaciones: una mirada innovadora. Santiago: Edición propia, 2008. p. 173-205.

BOENINGER KAUSEL, E. Políticas públicas en democracia: institucionalidad y experiencia chilena 1990-2006. Santiago: Uqbar, 2007.

CASTELLS, M.; HIMANEN, P. La sociedad de la información y el estado de bienestar. El modelo finlandés. Madrid: Alianza, 2002. CONFERENCIA IBEROAMERICANA DE ADMINISTRACIÓN PÚBLICA Y REFORMA DEL ESTADO. Carta Iberoamericana de Gobierno Electrónico. 2007. Disponible en: <http://old.clad.org/documentos/declaraciones/cartagobelec.pdf/view>. Acceso en: 10 mayo 2014.

CUNHA, M. A. V. C.; MIRANDA, P. R. M. O uso de TIC pelos governos: uma proposta de agenda de pesquisa a partir da produção acadêmica e da prática nacional. Organizações \& Sociedade, Salvador, v. 20, n. 66, p. 543-566, set. 2013. Disponible en: <http:// www.scielo.br/scielo.php?script=sci_arttext\&pid=S1984-92302013000300010\&lng=en\&nrm=iso>. Acceso en: 10 mayo 2014.

DAGNINO, R.; BRANDÃO, F. C.; NOVAES, H. T. Sobre o marco analítico-conceitual da tecnología social. In: FUNDAÇÃO BANCO DO BRASIL. Tecnologia Social: uma estratégia para o desenvolvimento. Rio de Janeiro: [s.n.], 2004. p. 15-64.

DE CERTEAU, M. La invención de lo cotidiano. Nueva ed. México, D.F.: Universidad Iberoamericana, 2007.

DETERDING, S. et al. Gamification: using game-design elements in non-gaming contexts. In: CHI CONFERENCE ON HUMAN FACTORS IN COMPUTING SYSTEMS, 2011, Vancouver. Proceeding... New York: ACM, 2011. p. 2425-2428.

FEENBERG, A. Critical Theory of Technology. New York: Oxford University Press, 1991.

Teoría crítica de la tecnología. Revista Iberoamericana de Ciencia, Tecnología y Sociedad, Buenos Aires, v. 2, n. 5, p. 109-123, mayo 2005.

. Transformar la tecnología: una nueva visita a la teoría crítica. Bernal: Univ. Nacional de Quilmes, 2012.

FOLADORI, G.; TOMMASINO, H. La solución técnica a los problemas ambientales. Revista Katálysis, Florianópolis, v. 15, n. 1, p. 79-83, jan./jun. 2012.

FREI TOLEDO, R. La cultura y el poder en las redes electrónicas. Enlace, Maracaibo, v. 4, n. 2, p. 29-41, ago. 2007.

GELMAN, C. R.; TOSONE, C. Teaching Social Workers to Harness Technology and Inter-Disciplinary Collaboration for Community Service. British Journal of Social Work, v. 40, n. 1, p. 226-238, 2010.

GIL-GARCIA, R.; LUNA-REYES, L. F. Integrating Conceptual Approaches to E-Government. In: KHOSROW-POUR, M. (Ed.). Encyclopedia of E-Commerce, E-Government, and Mobile Commerce. IGI Global, 2006, p. 637-638. Disponible en: <http://www.igiglobal.com/chapter/integrating-conceptual-approaches-government/12606>. Acceso en: 10 mayo 2014.

GILLINGHAM, P. Technology Configuring the User: Implications for the Redesign of Electronic Information Systems in Social Work. British Journal of Social Work, v. 46, n. 2, p. 323-338, dez. 2014.

GOLDKIND, L.; WOLF, L. A Digital Environment Approach: Four Technologies That Will Disrupt Social Work Practice. Social Work, v. 60, n. 1, p. 85-87, jan. 2015.

GUERRA, Y. A. D. Expressions of pragmatism in Social Work: preliminary reflections. Revista Katálysis, Florianópolis, v. 16, n. esp., p. 50-60, 2013. 
GUTIERREZ, L. Trabajo Social y tecnologías de la información y comunicación: Desafíos para la formación profesional. Revista Cuadernos de Trabajo Social, Madrid, v. 5, p. 41-54, 2012.

HEEKS, R.; BAILUR, S. Analyzing e-government research: Perspectives, philosophies, theories, methods, and practice. Government Information Quarterly, v. 24, n. 2, p. 243-265, apr. 2007.

HENMAN, P. Reconfiguring Power, Citizenship, Society. In: Governing Electronically: E-Government and the Reconfiguration of Public Administration, Policy and Power. [S.l.]: Palgrave Macmillan UK, 2010. p. 209-240.

LARRAÍN, J. Identidad chilena. Santiago: Lom, 2001.

MISHNA, F. et al. Here to Stay: Cyber Communication as a Complement in Social Work Practice. Families in Society-the Journal of Contemporary Social Services, v. 95, n. 3, p. 179-186, 2014.

NACIONES UNIDAS. Departamento de Asuntos Económicos y Sociales. UN Global E-Government Survey 2003. New York: Naciones Unidas, 2003. Disponible en: <http://unpan1.un.org/intradoc/groups/public/documents/un/unpan016066.pdf>. Acceso en: 10 mayo 2014.

Global E-Government Readiness Report 2004. Towards access for opportunity. New York: Naciones Unidas, 2004. Disponible en: <https://publicadministration.un.org/egovkb/portals/egovkb/Documents/un/2004-Survey/Complete-Survey.pdf>. Acceso en: 10 mayo 2014.

Global E-Government Readiness Report 2005. From E-Government to E-Inclusion. New York: Naciones Unidas, 2005. Disponible en: <http://unpan1.un.org/intradoc/groups/public/documents/un/unpan021888.pdf>. Acceso en: 10 mayo 2014. . UN E-Government Survey 2008. From E-Government to Connected Governance. New York: Naciones Unidas, 2008.

Disponible en: <http://unpan1.un.org/intradoc/groups/public/documents/un/unpan028607.pdf>. Acceso en: 10 mayo 2014.

. United Nations E-Government Survey 2010. Leveraging E-government at a Time of Financial and Economic Crisis. New York: Naciones Unidas, 2010. Disponible en: <http://unpan3.un.org/egovkb/global_reports/10report.htm>. Acceso en: 10 mayo 2014.

PROGRAMA DE LAS NACIONES UNIDAS PARA EL DESARROLLO. Las nuevas tecnologías: ¿un salto al futuro? Santiago: [s.n.], 2006.

RAUPP, F. M.; PINHO, J. A. G. Accountability em câmaras municipais: uma investigação em portais eletrônicos. Revista de Administração, São Paulo, v. 48, n. 4, p. 770-782, dez. 2013.

SANDOVAL-ALMAZAN, R.; GIL-GARCIA, J. R. Are government internet portals evolving towards more interaction, participation, and collaboration? Revisiting the rhetoric of e-government among municipalities. Government Information Quarterly, v. 29, supplement 1, p. S72-S81, jan. 2012.

SANTOS, B. Para un nuevo sentido común: La ciencia, el derecho y la política en la transición paradigmática. In: Crítica de la Razón Indolente: contra el desperdicio de la experiencia. Bilbao: Desclée de Brouwer, 2003. Volumen 1.

THOMAS, H. De las tecnologías apropiadas a las tecnologías sociales. conceptos / estrategia / diseños / acciones. 2009a. Disponible en: <http://studylib.es/doc/4701331/de-las-tecnolog\%C3\%ADas-apropiadas-a-las-tecnolog\%C3\%ADas-sociales.pdf>. Acceso en: 10 mayo 2014.

. Sistemas tecnológicos sociales y ciudadanía socio-técnica. Innovación, desarrollo y democracia. In: TULA, F.; GIULIANO, G. (Ed.). Culturas Científicas y Alternativas Tecnológicas. Buenos Aires: Ministerio de Ciencia, Tecnología e Innovación Productiva de la Nación, 2009b. p. 65-86.

VIVERO, L. Cambios sociopolíticos en América Latina: Desafíos para un trabajo social crítico latinoamericano. Eleuthera, Manizales, v. 6, p. 15-25, 2012.

YILDIZ, M.; SAYLAM, A. E-government discourses: An inductive analysis. Government Information Quarterly, v. 30, n. 2, p. 141153, apr. 2013.

XIA, F. et al. Internet of Things. International Journal of Communication Systems, v. 25, n. 9, sept. 2012.

\section{Notas}

1 Es importante precisar que Henman (2010) usa el concepto de táctica para referirse a lo que Feenberg (2012) y De Certeau (2007) han denominado como decisiones institucionales estratégicamente organizadas. Para estos autores, la táctica es la respuesta adaptativa de quienes no tienen poder formal.

2 Según señalan Xia et al. (2012, p. 1101), la internet of things se refiere a: “[...] la conexión en red de objetos cotidianos, que son equipados con inteligencia ubicua [...]”. Con ello se posibilita la interacción virtual y presencial con objetos.

3 La gamification se refiere a la utilización de dinámicas y elementos de los videojuegos para adaptar y mejorar servicios y aplicaciones que no se vinculan con el juego (DETERDING et al., 2011).

\section{Dr. Helder Binimelis-Espinoza}

hbinimelis@uct.cl 
Doctorado en Investigación en Ciencias Sociales con Mención en Sociología por la Facultad Latinoamericana de Ciencias Sociales (FLACSO)

Profesor del Departamento de Trabajo Social de la Universidad Católica de Temuco (UCT)

\section{UCT}

Manuel Montt, 56 - Edificio C, Oficina 503

Campus San Francisco, Departamento de Trabajo Social

Temuco - Araucanía - Chile 